\title{
Hard X-ray emission from a flare-related jet
}

\author{
H. M. Bain and L. Fletcher
}

Department of Physics and Astronomy, University of Glasgow, Glasgow, G12 8QQ, UK

e-mail: hmbain@astro.gla.ac.uk

Received 17 Febuary 2009 / Accepted 24 September 2009

\section{ABSTRACT}

\begin{abstract}
Aims. We aim to understand the physical conditions in a jet event which occurred on the 22nd of August 2002, paying particular attention to evidence for non-thermal electrons in the jet material.

Methods. We investigate the flare impulsive phase using multiwavelength observations from the Transition Region and Coronal Explorer (TRACE) and the Reuven Ramaty High Energy Spectroscopic Imager (RHESSI) satellite missions, and the ground-based Nobeyama Radioheliograph (NoRH) and Radio Polarimeters (NoRP).

Results. We report what we believe to be the first observation of hard X-ray emission formed in a coronal jet. We present radio observations which confirm the presence of non-thermal electrons present in the jet at this time. The evolution of the event is best compared with the magnetic reconnection jet model in which emerging magnetic field interacts with the pre-existing coronal field. We calculate an apparent jet velocity of $\sim 500 \mathrm{~km} \mathrm{~s}^{-1}$ which is consistent with model predictions for jet material accelerated by the $\boldsymbol{J} \times \boldsymbol{B}$ force resulting in a jet velocity of the order of the Alfvén speed $\left(\sim 100-1000 \mathrm{~km} \mathrm{~s}^{-1}\right)$.
\end{abstract}

Key words. Sun: activity - Sun: flares - Sun: X-rays, gamma rays - Sun: radio radiation

\section{Introduction}

Solar X-ray jets (transient bursts of collimated flows of plasma) were first observed by the Yohkoh Solar X-ray Telescope (SXT: Tsuneta et al. 1991) (Shibata et al. 1992; Strong et al. 1992). It has been shown that jets are spatially correlated with active regions, $\mathrm{X}$-ray bright points and regions of emerging flux and can be associated with small footpoint flares (Shibata et al. 1992, 1994). Several studies of X-ray jets (Shimojo et al. 1996; Canfield et al. 1996) yielded characteristic properties such as length and width observed to be in the range of a few $10^{4}-10^{5} \mathrm{~km}$ and $5 \times 10^{3}-10^{5} \mathrm{~km}$ respectively. Apparent jet velocities of $10-1000 \mathrm{~km} \mathrm{~s}^{-1}$ (average $\sim 200 \mathrm{~km} \mathrm{~s}^{-1}$ ) are observed and the kinetic energy of the jet is thought to be $10^{25}-10^{28} \mathrm{erg}$. Shimojo \& Shibata (2000) analysed a number of jets and their footpoint flares finding values for jet temperature and density of 3-8 MK (average 5.6 MK) and 0.7-4.0 × $10^{9} \mathrm{~cm}^{-3}$ (average $\left.1.7 \times 10^{9} \mathrm{~cm}^{-3}\right)$ respectively. More recently observations of solar X-ray jets have been made using data from the Hinode X-ray Telescope (XRT: Golub et al. 2007) (Shimojo et al. 2007; Chifor et al. 2008; Savcheva et al. 2007). The results are generally consistent with the previous studies however XRT's improved spatial and temporal resolution has revealed many smaller X-ray jet events.

It has been observed that in general there are two kinds of jet; 1) the anemone-shaped jet, consistent with emerging photospheric field interacting with pre-existing coronal field which is vertical or oblique; 2) the two-sided-loop type where the interaction occurs with overlying horizontal coronal magnetic field (Shimojo et al. 1996). Observational properties such as the jet velocity are an important diagnostic of the jet acceleration mechanism. Several jet models have been suggested, most models at some stage invoke magnetic reconnection as the source of a rapid injection of energy. The magnetic reconnection jet model (Heyvaerts et al. 1977; Shimojo et al. 1996; Shimojo \& Shibata 2000) describes a bundle of emerging photospheric field reconnecting with a pre-existing overlying coronal magnetic field. As the result of the reconnection, surrounding plasma is heated to X-ray emitting temperatures and subsequently ejected out along the direction of the reconnected field. Plasma propagating downwards forms X-ray emitting loops at the foot of the jet. Observations have shown that $\mathrm{H} \alpha$ surges are often associated with X-ray jets (Canfield et al. 1996). In the reconnection model this is as a result of a "magnetic sling-shot" effect caused by magnetic tension as the previously highly stressed emerging field straightens out. This results in the ejection of cool plasma $(10000 \mathrm{~K})$ that had been supported by the field. Magnetohydrodynamic simulations of this scenario describe the observations well (Yokoyama \& Shibata 1995; Nishizuka et al. 2008; Moreno-Insertis et al. 2008). Ejected jet material accelerated by the $\boldsymbol{J} \times \boldsymbol{B}$ force will have a velocity of the order of the Alfvén speed.

There have been observations of jets with a helical twisted shape. The "magnetic twist" model explains this as the result of reconnection between twisted and untwisted magnetic field (Shibata \& Uchida 1986; Shibata et al. 1992; Alexander \& Fletcher 1999; Pariat et al. 2009). The ejected material is accelerated by the $\boldsymbol{J} \times \boldsymbol{B}$ force as the twisted magnetic field relaxes. As with the magnetic reconnection model, the ejected material will have a velocity of the order of the Alfvén speed. Magnetic islands of cool plasma form within the twisted field, described by the "melon-seed" model for spicules (Uchida 1969), which propagate out as the field relaxes. This also occurs at the Alfvén speed.

Other models suggest that the acceleration mechanism is related to gas pressure. Evaporation flows occur as the result of a rapid release of energy in the corona. In this situation the jet velocity will be of the order of the sound speed (Shibata et al. 1992; Sterling et al. 1993)

Type III radio bursts, caused by unstable beams of accelerated electrons, are often associated with jets. Electrons accelerated in association with jets have been detected in space 
(Christe et al. 2008). Another diagnostic of fast electrons is bremsstrahlung X-ray emission which can be observed with the Reuven Ramaty High Energy Spectroscopic Imager, (RHESSI: Lin et al. 2002). However until now no evidence of hard X-ray emission has been observed directly from the jet in the corona. This could be because it is rare to find a coronal jet dense enough to provide a bremsstrahlung target for the electrons, or hot enough to generate high energy thermal emission. In this paper we report what we believe to be the first observation of hard X-ray emission formed in a coronal jet. We observe a flare-related jet which occurred on the 22nd August 2002. Observations from the Transition Region Coronal Explorer, (TRACE: Handy et al. 1999) and RHESSI satellite missions are presented. The event was also observed by the ground-based Nobeyama Radioheliograph (NoRH: Nakajima et al. 1994) and Polarimeters (NoRP: Torii et al. 1979). Together these observations cover the X-ray, extreme ultraviolet (EUV) and radio regimes.

\section{Event overview}

The jet occurred on the 22nd of August 2002 preceding a GOES M5.4 class flare. Time profiles from GOES, RHESSI, TRACE and NoRH can be seen in Fig. 1. Vertical dashed lines represent $32 \mathrm{~s}$ time intervals used for RHESSI imaging (see Fig. 5 later). At 01:40 GOES, RHESSI and NoRH $17 \mathrm{GHz}$ show an increase in the signal above background levels. This is due to a small source slightly north of the jet region, centred at $\left(825^{\prime \prime},-225^{\prime \prime}\right)$ which does not appear to be related to the jet. The evolution of the event can be followed best using high resolution TRACE observations (see Fig. 2), only available for the $195 \AA$ passband during the event. These images have a time resolution of $\sim 9 \mathrm{~s}$ and a spatial resolution of $1^{\prime \prime}$. Dark black areas show regions of saturation. This is particularly noticeable later in the event when the hot loops form. The high contrast is to allow faint features to be visible. Under normal active region conditions this wavelength is dominated by Fe XII line emission at $1.4 \times 10^{6} \mathrm{~K}$. During flares it will be dominated by higher temperature plasma, providing both Fe XXIV and Ca XVII line emissions $\left(5 \times 10^{6} \mathrm{~K}\right.$ and $\left.(1-2) \times 10^{7} \mathrm{~K}\right)$ and a significant thermal continuum due to free-free and free-bound emission, which is in fact dominant at temperatures of around $10^{7} \mathrm{~K}$ (Feldman et al. 1999). The images were exposure normalised using the Solarsoft routine trace_prep. Cosmic ray spikes were also removed using this routine.

Preflare activity can be seen in the region as early as $\sim 01: 25$ (Fig. 2a). From 01:27 a complex system of twisted loops begin to brighten (Figs. 2b-2f) and appears to rise. At 01:35 part of this system becomes twisted in a configuration similar to that found in models of the kink instability (Török \& Kliem 2005). This can be seen best a few minutes later when it is at its brightest in Fig. 2e (see arrow). Although faint, this feature is present right up until the main ejection. To the north-west, at the right foot of the twisted field $\left(820^{\prime \prime},-270^{\prime \prime}\right)$, faint material starts to move outward, starting at 01:31, along a separate large scale loop or possibly even open interplanetary field. This is seen at its brightest in Fig. 2d (see arrow). Small amounts of faint material continues to move in this direction for several minutes before the main ejection, becoming more obvious at 01:42 (see Figs. 2e and 2f). Unfortunately a gap in the TRACE data between $01: 45: 49$ and 01:49:44 prevents us following this material with TRACE. For the purpose of understanding this complex event, we label pre-jet activity, including the ejection missed by TRACE up to 01:49:44, as "pre-gap".

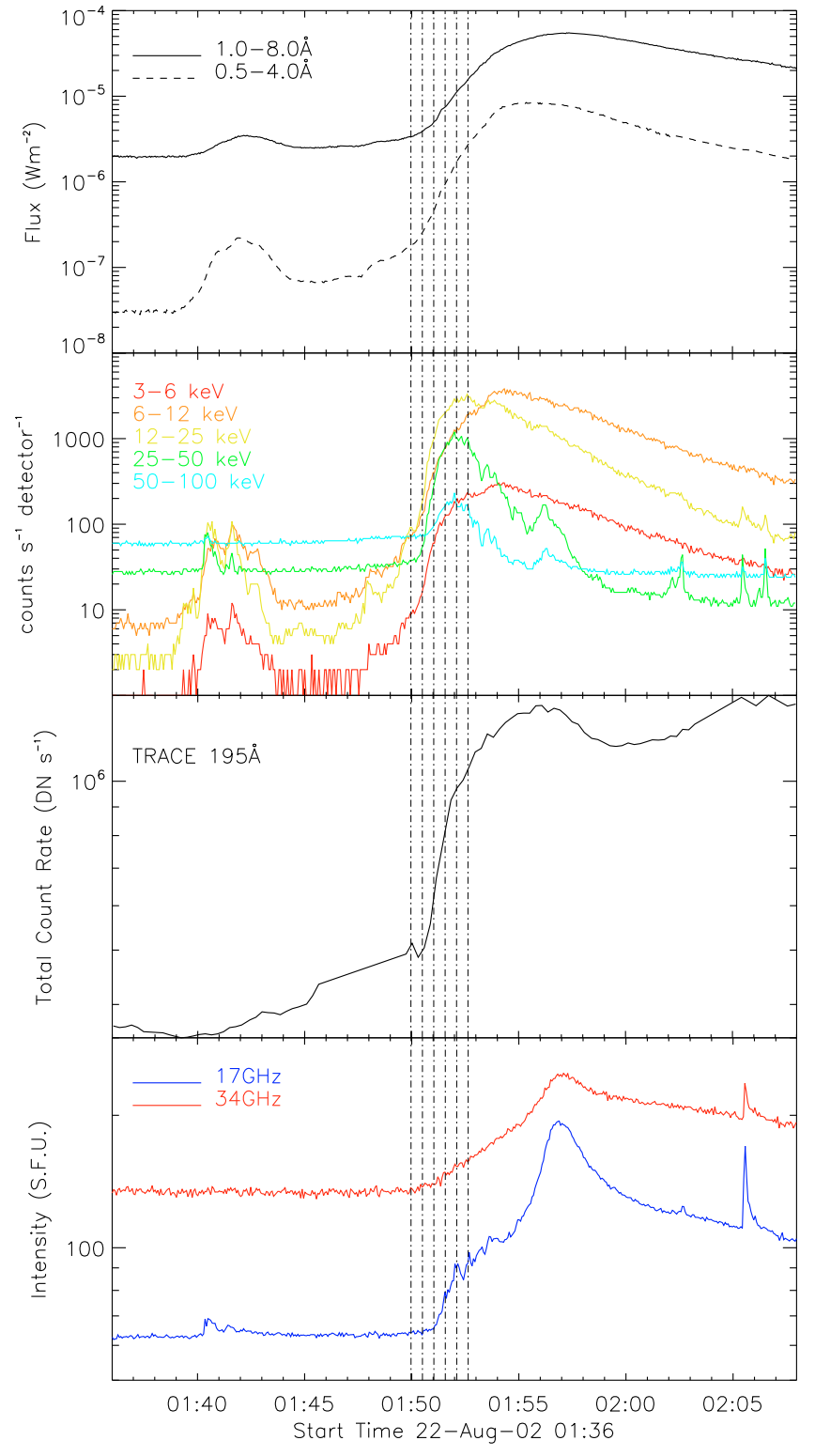

Fig. 1. From top to bottom: GOES, RHESSI, TRACE and NoRH lightcurves. Vertical dashed lines represent $32 \mathrm{~s}$ time intervals used for RHESSI imaging (see Fig. 5). (See electronic version for colour plots.)

During the data gap increased amounts of material are ejected. This can be seen, in images immediately after the data gap, as plasma at greater heights in the corona (Figs. $2 \mathrm{~g}$ and $2 \mathrm{~h}$ ). Also from the images immediately after the data gap (Figs. $2 \mathrm{~g}$ and $2 \mathrm{~h}$ ) we can see that the rising twisted loops appear to have opened to the right of the kink feature, which is still visible (see arrow in Fig. 2h). This results in a large amount of plasma being ejected, beginning at $\sim 01: 50: 35$. The feature to the left of the jet can then be seen to straighten out. The ejected material appears to untwist slightly as the twist from the emerging field is transferred to the "open" field lines of the jet as a result of reconnection. The material then passes out of the TRACE field of view after $\sim 01: 52$.

Material continues to be ejected until 02:09 (Figs. 2g-2m). The total duration of jetting material is therefore around $40 \mathrm{~min}$. Hot loops can be seen forming between EUV ribbons, roughly $\left(820^{\prime \prime},-260^{\prime \prime}\right)$, as early as $\sim 01: 51: 30$. These loops brighten, expand and then fade away over the course of an hour 


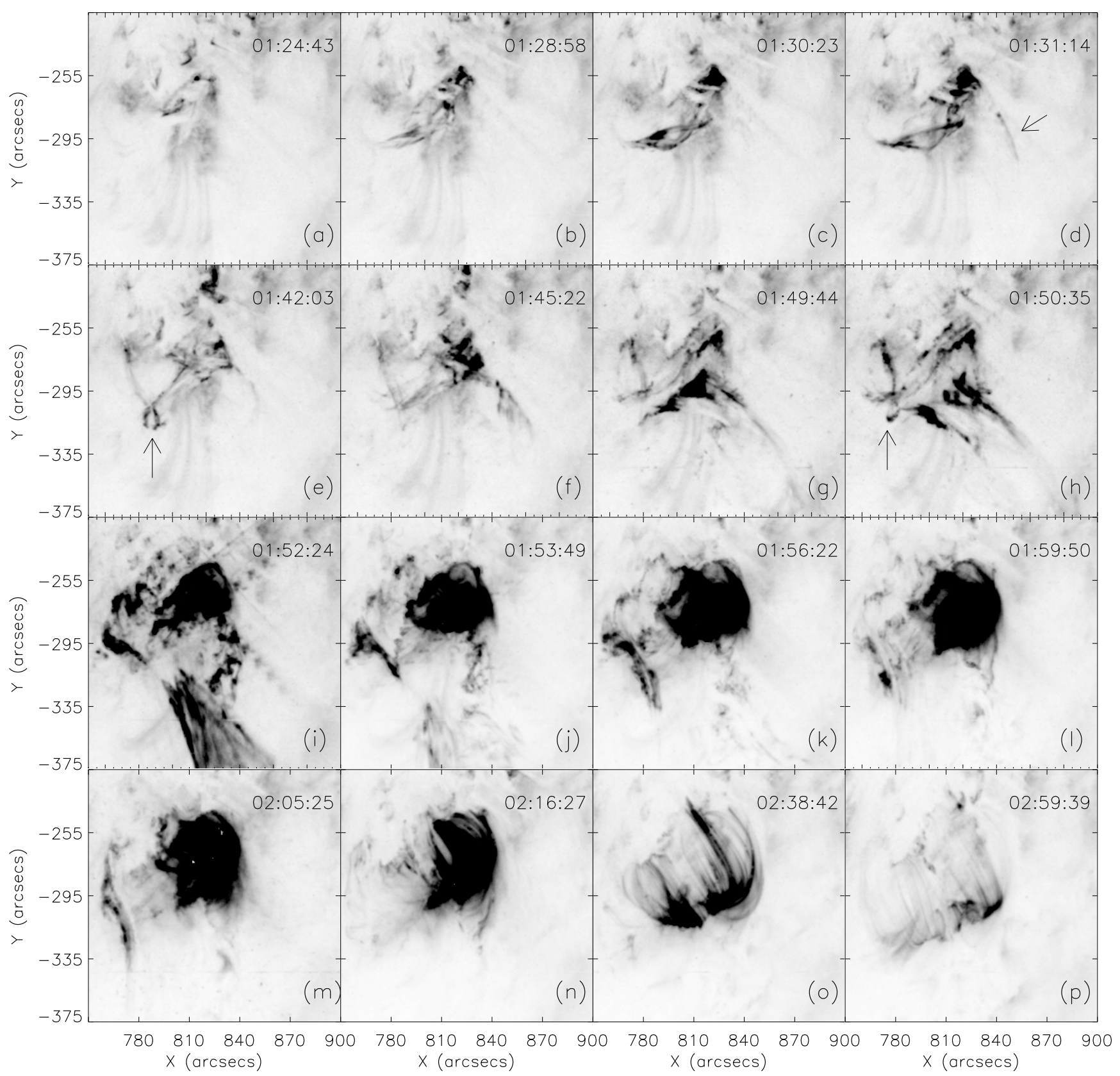

Fig. 2. TRACE $195 \AA$ images for selected times throughout the event showing the evolution of the jet. These images have a time resolution of $\sim 9 \mathrm{~s}$ and a spatial resolution of $1^{\prime \prime}$.

(Figs. 2n-2p). Activity from 01:49:44 onwards, including the main ejection, is labelled "post-gap". In this paper we concentrate on the impulsive and rise phase of the event i.e. post-gap times when the main ejection of material occurs.

Due to the jet extending beyond the TRACE field of view we were unable to determine its full length. However an estimate of jet width was found to be $(2.32 \pm 0.2) \times 10^{4} \mathrm{~km}$. This was determined by eye and assigned an error of \pm 5 pixels. To calculate the apparent velocity of the jet during the main ejection a timeslice image was created using post-gap images (Fig. 3). These images were rotated such that the ejected material moves in a vertical direction. A subregion, through which the ejected material moves, was then chosen, shown by dashed lines on the inserted TRACE image in Fig. 3. Note that the TRACE insert is cut off at the top right corner due to the edge of the TRACE field of view. For each image, intensity was summed over rows to give a 1D array of intensity as a function of height. These were then combined to make Fig. 3, showing how the jet intensity varies in height as a function of time. The faint front edge of the jet was determined to be at the $5 \%$ level of the maximum timeslice intensity (asterisks). By fitting these points we obtain an apparent jet velocity of $540 \pm 45 \mathrm{~km} \mathrm{~s}^{-1}$ (solid line Fig. 3). It should be noted that this is the apparent jet velocity, there will be some degree of uncertainty due to the material following a curved trajectory and also due to the overall projection effects. However this method provides a ball-park figure which is consistent with previous jet studies and is on the order of the Alfvén velocity in the corona in accordance with a jet accelerated by the magnetic $\boldsymbol{J} \times \boldsymbol{B}$ force. To further check this value a source of brightly emitting plasma was followed by eye throughout the main ejection (diamonds). Fitting points 1 to 4 we obtain a value of $493 \pm 96 \mathrm{~km} \mathrm{~s}^{-1}$ (dashed line Fig. 3). Here the 5th point has 


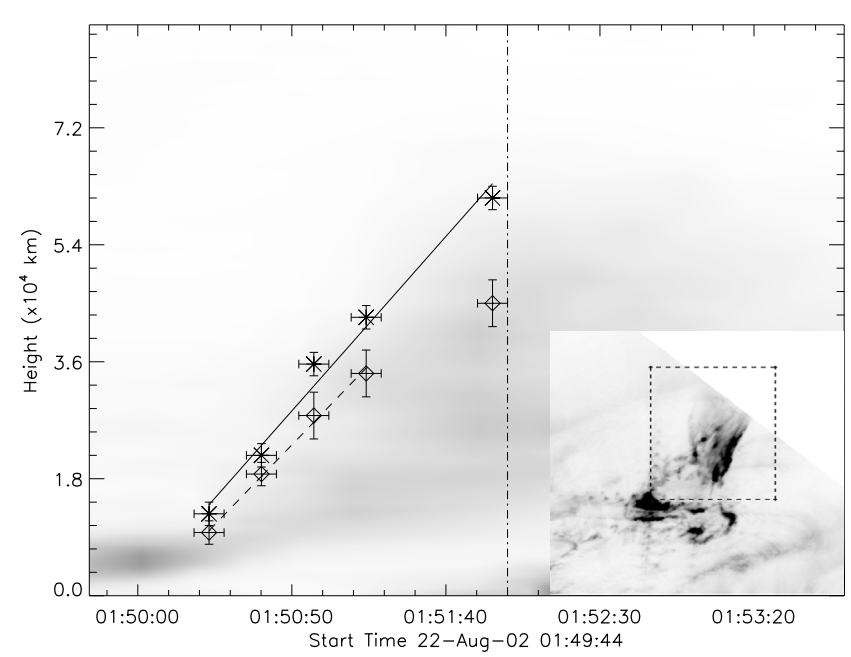

Fig. 3. Velocity timeslice showing jet intensity as a function of height and time. Vertical dash-dot line shows the time at which the jet emission passes out of the TRACE field of view. Dashed lines on insert image show the region used for the analysis. Asterisks and solid line shows a fit to the faint front edge of the jet at the $5 \%$ level. Diamonds and dashed line shows a fit to points determined from tracking a brightly emitting source of plasma by eye.

not been included in the fit as at the time of the image the material appeared to follow field that curved away from the vertical and our technique thus underestimates the distance travelled.

\section{Hard X-ray observations}

\subsection{RHESSI imaging}

RHESSI is a single instrument capable of imaging and spectroscopy of photons of energies ranging from $3 \mathrm{keV}$ (soft X-rays) to $17 \mathrm{MeV}$ ( $\gamma$-rays), with spatial resolution of $2.3^{\prime \prime}(\approx 1660 \mathrm{~km})$ and spectral resolution of $\approx 1-10 \mathrm{keV} \mathrm{FWHM}$. The instrument consists of 9 bi-grid rotating modulation collimators each sampling a different spatial scale. Cryogenically-cooled germanium detectors record each photon's arrival time and energy.

Unlike for most large flares the RHESSI data for this period was not interrupted by movements of the spacecrafts attenuators. Note the RHESSI thin attenuator (A1) is in place throughout the entire event. A series of 32 s RHESSI images (times shown as vertical dashed lines in Fig. 1) were reconstructed using detectors 3 to 9 providing a spatial resolution of $\sim 7^{\prime \prime}$. Energy bins of 6-12 keV, 12-20 keV, 20-30 keV and 30-50 keV were used. It is hoped that by using these energy bands it will be possible to distinguish sources of thermal and non-thermal emission. A more detailed RHESSI lightcurve is shown in Fig. 4. Here the lightcurve is split into 10 logarithmic energy bins between $3 \mathrm{keV}$ and $100 \mathrm{keV}$. Looking at the trend of the lightcurve in each energy band we can see that the emission above $\sim 17 \mathrm{keV}$ follows a very similar profile to that of higher energies suggesting that the emission is non-thermal in nature above this energy. RHESSI spectroscopy (see Sect. 3.2) also confirms the turnover between thermal and non-thermal emission to be $\sim 20 \mathrm{keV}$. Therefore we consider the $20-30$ and $30-50 \mathrm{keV}$ bands to be free of thermal emission during these time intervals, to a first approximation.

Figure 5 shows RHESSI image contours overlaid on the corresponding TRACE images. Rows show times 01:48:58, 01:50:30, 01:51:02, 01:51:34 and 01:52:06 and columns show contours of RHESSI energy bands at 6-12 (blue), 12-20 (green), 20-30 (magenta) and 30-50 keV (red). The far right column

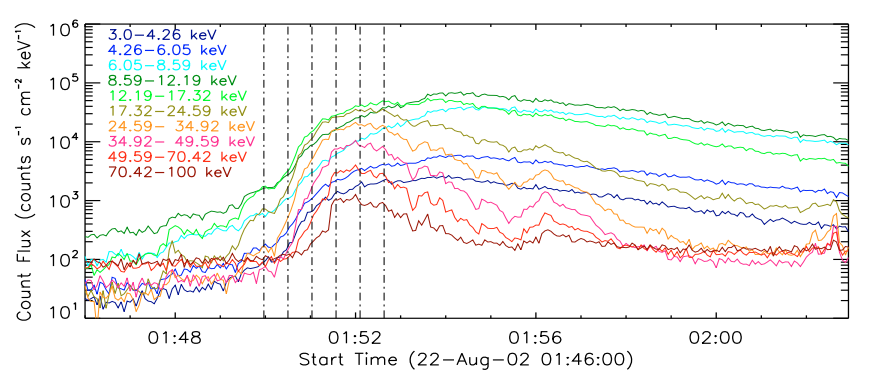

Fig. 4. RHESSI lightcurve with logarithmic energy binning. Vertical dashed lines represent $32 \mathrm{~s}$ time intervals used for RHESSI imaging (see Fig. 5). (See electronic version for colour plots.)

shows all RHESSI contours overlaid. TRACE pointing is controlled by an Image Stabilization System (ISS) which is accurate to roughly $5^{\prime \prime}-10^{\prime \prime}$. A pointing drift on the order of $\pm 1^{\prime \prime}$ as a result of temperature fluctuations can cause some additional offset (Aschwanden et al. 2000). Correction to the alignment can be done using image cross correlation with other instruments. However for this event no TRACE pointing correction was carried out. From the images in Fig. 5 it is clear that the RHESSI contours align well with bright TRACE features.

From row 2 of Fig. 5, at time 01:50:30 (hereafter interval 2), emission as high as the RHESSI 30-50 keV energy band can be seen to be co-spatial with the jet around $\left(820^{\prime \prime},-300^{\prime \prime}\right)$. From the TRACE images this is the region in which we think the twisted magnetic field became unstable, resulting in the ejection of plasma. These observations indicate the presence of hard $\mathrm{X}$-rays and hence non-thermal electrons in the jet, and we believe this to be the first observation of its kind. Although not shown here, RHESSI images at these energies with better time resolution have been studied to see how this source evolves, and on this basis the question of whether the emission could be from a coronal loop can been dismissed: the hard X-ray jet emission can be seen to propagate out on a similar time scale to the ejected material and does not behave like a coronal loop. In general coronal loops are not present in the early impulsive phase of the event.

As the event continues (rows 3, 4 and 5 of Fig. 5) compact footpoint sources are seen forming at higher energies from 20-50 keV. In the low energy bands sources begin to form, cospatial with the hot loops faintly visible in TRACE (see also Fig. 2 (i) onwards), seen as the right hand source in Fig. 5 rows 4 and 5 at roughly $\left(820^{\prime \prime},-260^{\prime \prime}\right)$. Note that although these sources appear to be cospatial with the higher energy sources this is thought to be just a projection effect. For this paper we concentrate primarily on the emission present in the jet at 01:50:30.

\subsection{RHESSI spectroscopy}

Figure 6 shows the fitted RHESSI spectra obtained from detector 3 for time interval 2. Energy bins of $0.33 \mathrm{keV}$ at lower energies $(6-12 \mathrm{keV})$ were used to properly fit the iron and nickel lines at 6.7 and $8.0 \mathrm{keV}$. Above $12 \mathrm{keV}$ energy binning of $1 \mathrm{keV}$ was used. The black line shows the background subtracted data and grey the background emission. Figure 6 shows two possible fits to the data between $6-100 \mathrm{keV}$. Fit 1 is an isothermal (green solid) plus thin target (blue solid) fit to the electron spectrum. Fit 2 is an isothermal (green dashed) plus thick target (blue dashed) fit. The thermal component is characterised by a temperature and an emission measure. The thick and thin target fit 


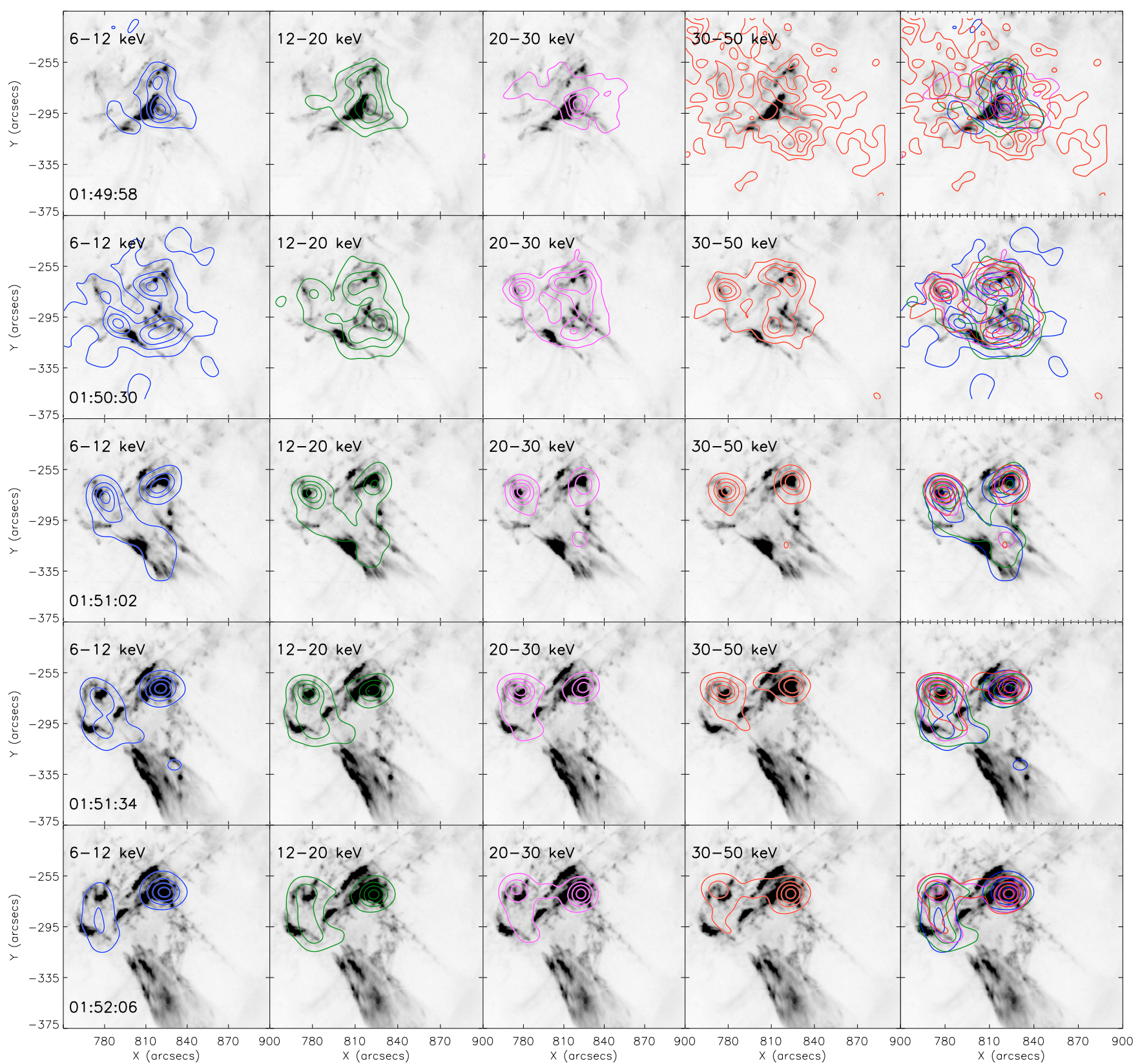

Fig. 5. TRACE images with RHESSI image contours overlaid. Rows show time slices at times 01:48:58, 01:50:30, 01:51:02, 01:51:34 and 01:52:06 and columns show contours of RHESSI energy bands at 6-12 (blue), 12-20 (green), 20-30 (magenta) and 30-50 keV (red). The far right column shows all RHESSI contours overlaid. Contour levels are at 90, 75, 50 and $25 \%$.

functions are characterised by a power law fit to non-thermal electrons between an electron low and a high energy cut off. The electron spectral index and low energy cut off were allowed to vary. Figure 6 shows the conversion to photon spectra and values of temperature, photon spectral index and electron low energy cut off are stated in each plot. An additional Gaussian line (orange) was added to fit the line feature seen at $11 \mathrm{keV}$. It is thought that this is an instrumental feature but it is not fully understood what is producing this (Dennis 2009, private communication). Red shows the overall combination of fitted functions. Spectral fitting was carried out for individual detectors 1, 3, 4, 6,8 , and 9 and average parameters values calculated. The thermal component is characterised by a temperature of $24 \mathrm{MK}$ and $26 \mathrm{MK}$ for fit 1 and 2 respectively. The hard X-ray spectral index obtained was 4.67 and 4.77 for fit 1 and 2 respectively, thus supporting the evidence for non-thermal electrons present in the event. However using only spatially integrated spectra it is not possible to separate the contribution from the jet and the footpoints and hence it is impossible to determine the spectral parameters resulting from the jet alone, therefore we have carried out imaging spectroscopy.

To perform imaging spectroscopy for time interval 2, images were made using pseudo-logarithmic energy binning from $7.5 \mathrm{keV}$ to $48.5 \mathrm{keV}$. Despite summing counts from detectors 1 , $3,4,6,8$ and 9 there were not enough counts for image synthesis above this energy to allow fitting to higher energies. Figure 7 (top left) shows a RHESSI image at 29.5-37.5 keV. Marked on the image are the regions chosen for individual spectral analysis to separate the emission from the jet and the footpoints. Here we fit an isothermal plus broken power fit components to the 


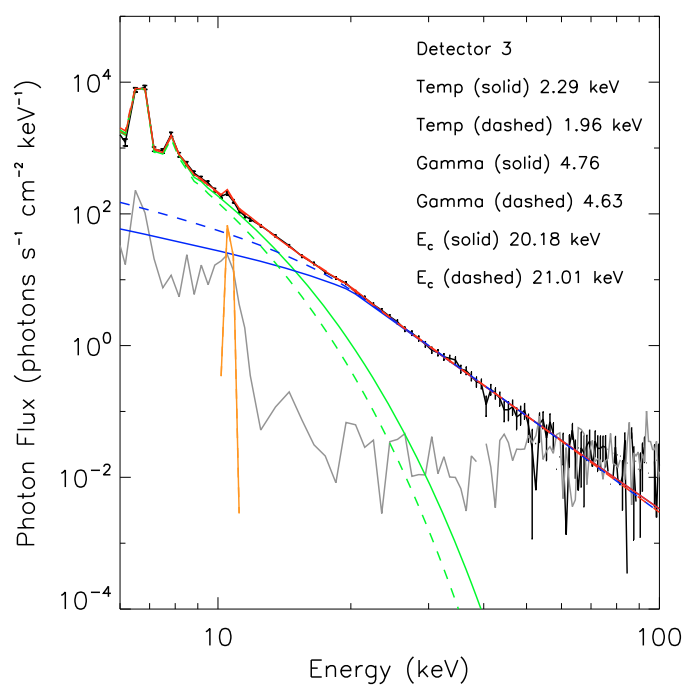

Fig. 6. RHESSI photon spectra obtained from detector 3 for time interval 2. Black lines shows the background subtracted data and grey shows the background. The plot shows two possible fits, isothermal plus thin target (solid lines) and isothermal plus thick target (dashed lines). Green lines show isothermal fits. Blue lines show thick and thin target fits. Red shows the combined fit functions. Orange shows a Gaussian line added to fit a feature seen at $11 \mathrm{keV}$.
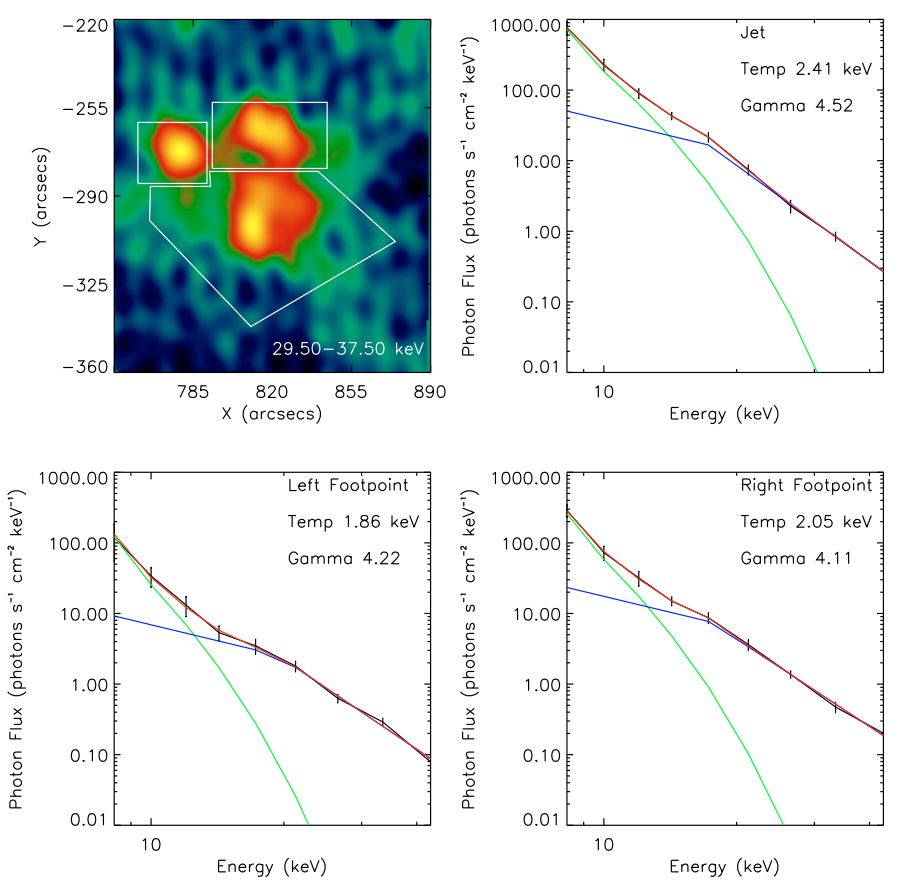

Fig. 7. RHESSI image at $29.5-37.5 \mathrm{keV}$ (top left) showing regions chosen for individual spectral analysis. Imaging spectroscopy results for the jet (top right), left footpoint (bottom left) and right footpoint (bottom right). Black lines show the observed photon spectra, green and blue show the isothermal and thick target fit functions respectively. Red shows the combination of fitted functions.

photon spectrum. For the broken power law component the spectral index below a variable break energy is fixed at 1.5. Above the break the spectral index is allowed to vary. The results are shown in Fig. 7. Black lines show the observed photon spectra, green and blue show the isothermal and broken power law fit functions respectively. Red shows the combination of fitted functions. Stated on each plot are the values obtained for temperature and photon spectral index. We draw particular attention to Fig. 7 (top right) which shows the fitted photon spectrum for the jet characterised by a power law with a spectral index of 4.5 (slightly softer than the value obtained for the footpoints) confirming a non-thermal hard X-ray component in the jet, in turn implying the presence of non-thermal electrons. An estimate of jet temperature was found to be $28 \mathrm{MK}$. Temperature estimates for the hard X-ray footpoints are stated in each plot. However it should be noted that thermal footpoint emission could be subject to contamination as a result of the bright coronal source contributing to the flux. This is due to side lobes (Krucker \& Lin 2002).

\section{Radio observations}

\subsection{Nobeyama Radioheliograph}

The Nobeyama Radioheliograph, a ground-based solardedicated radio interferometer, records full disk images of flux density (brightness) at 17 and $34 \mathrm{GHz}$ microwave frequencies with spatial resolution of $10^{\prime \prime}$ and $5^{\prime \prime}$ respectively. During flare events NoRH has an excellent temporal resolution of $0.1 \mathrm{~s}$. Measurements of polarisation are also made at $17 \mathrm{GHz}$. At these frequencies the primary solar emission mechanism is gyrosynchrotron.

Images at $17 \mathrm{GHz}$ (top) and $34 \mathrm{GHz}$ (bottom) are shown in Fig. 8 for the same time intervals as with RHESSI. Note the field of view here is larger than that of Fig. 5 and shows an active region to the north-west of the jet. As time progresses, ejected material can be seen moving southwest away from the footpoint region. From the flux at $17 \mathrm{GHz}$ and $34 \mathrm{GHz}$ it is possible to determine a radio spectral index $\alpha$ from $\mathrm{F}(v) \sim v^{\alpha}$, where $\mathrm{F}(v)$ is the flux at frequency $v$. Overlaid on the NoRH images are contours of spectral index $\alpha$. White lines show positive spectral index from 0 to 2 in steps of 0.5 , corresponding to optically thick emission. Black lines show lines of negative spectral index from -1 to -4 in steps of 1 corresponding to optically thin emission. As the event progresses footpoints can be seen forming. At 01:51:34 and onwards these regions become optically thin. Confirmation of this can be see in Fig. 11 which shows spectra from the Nobeyama Polarimeters from 01:51 onwards. The turnover in the spectrum occurs at lower frequencies than those observed by NoRH indicating optically thin emission at $17 \mathrm{GHz}$ and $34 \mathrm{GHz}$. See Sect. 4.2 for more details. At these frequencies we can assume that the microwave emission is gyrosynchrotron radiation from particles at even higher energies than those observed via RHESSI hard X-rays, and hence definitely non-thermal in nature.

We pay particular attention to the images at 01:50:30 (see Fig. 9). On the left the contours of NoRH $17 \mathrm{GHz}$ (dot-dashed black), $34 \mathrm{GHz}$ (solid blue) and RHESSI 30-50 keV (dashed red) are overlaid on TRACE. It is important to note that NoRH images are subject to pointing errors due to random fluctuations of the Earth's ionosphere, particularly during a flare. Observing the left footpoint at $17 \mathrm{GHz}$ and $30-50 \mathrm{keV}$ suggests a displacement of $10^{\prime \prime}$ in the E-W direction. Despite this the emission seen by NoRH and RHESSI are well correlated with each other and with the EUV jet supporting the evidence for non-thermal particles in the jet at this time. Figure 9 (right) shows a contour map of spectral index $\alpha$ with contours of positive $\alpha$, ranging from 0 to 1.5 in steps of 0.25 . The value of $\alpha$ for the jetting region is 


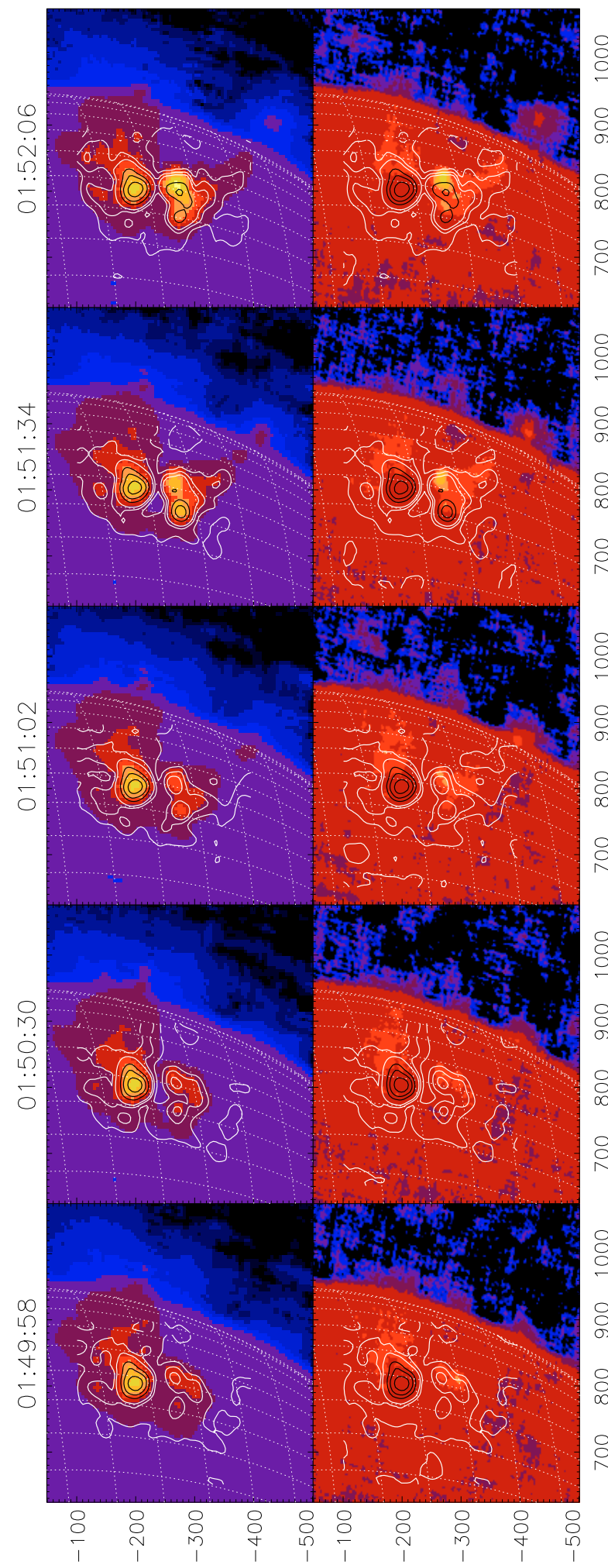

Fig. 8. NoRH $17 \mathrm{GHz}$ (top) and $34 \mathrm{GHz}$ (bottom) images at time intervals used for RHESSI imaging. White lines show positive spectral index from 0.5 to 1.5 in steps of 0.5 corresponding to optically thick plasma. Black lines show lines of negative spectral index from -1 to -4 in steps of 1 corresponding to optically thin plasma. (See electronic version for colour plots.)

$\sim 0.75$ corresponding to optically thick emission from the fastmoving electrons.

\subsection{Nobeyama polarimeters}

In addition to data from the Nobeyama Radioheliograph, radio flux and polarisation at 1, 2, 3.75, 9.4, 17, 34 and $80 \mathrm{GHz}$ frequencies can be obtained from the Nobeyama Polarimeters (NoRP). For this event the flux at $80 \mathrm{GHz}$ remained at the background level and was excluded. Figure 10 (top) shows a plot of total flux $(\mathrm{R}+\mathrm{L})$ and Fig. 10 (middle) shows the degree of circular polarisation (R-L), R and L denote the right and left circular polarisation. Note that NoRP records flux from the full disk, however as there appears to be no other significant activity on the disk during this time interval, the emission recorded is likely to be from the jet event. It is interesting to note that the spiky bursts between 01:51:00 and 01:52:00 seen at the lower frequencies during the impulsive phase have no counterpart at higher radio frequencies. At $1 \mathrm{GHz}$ these bursts are thought to be due to plasma emission at the local plasma frequency and its harmonics caused by a beam of electrons producing Langmuir waves due to a bump-in-tail instability. The non-linear nature of Langmuir wave-particle interactions results in the increased flux at $1 \mathrm{GHz}$. This effect will also be reflected in the brightness temperature $T_{\mathrm{b}}$, which can be calculated using the Rayleigh-Jeans law (Eq. (1)) where $k_{\mathrm{b}}$ is Boltzmann's constant (Dulk 1985).

$$
\begin{aligned}
B_{v} & =\frac{2 k_{\mathrm{b}} T_{\mathrm{b}} v^{2}}{c^{2}} \\
S_{v} & =\int_{\text {source }} B_{v}(\theta, \phi) P_{n}(\theta, \phi) \mathrm{d} \Omega .
\end{aligned}
$$

Rearranging Eq. (2) where $S_{v}$ is the spectral flux density received by NoRP, $B_{v}(\theta, \phi)$ is the surface brightness for a source subtending $\mathrm{d} \Omega . P_{n}(\theta, \phi)$ is the antenna power pattern. For sufficiently small source size, compared with the NoRP beam size, we can set $P_{n}(\theta, \phi) \simeq 1$ across the source. By subtracting a background flux we assume that emission observed results from an emitting source size $\mathrm{d} \Omega$, taken to be $50^{\prime \prime} \times 100^{\prime \prime}$, roughly the extent of the jet region visible from NoRH images at 17 and $34 \mathrm{GHz}$ thus an upper limit of $\mathrm{d} \Omega$. We obtain an estimate for the brightness temperature $T_{\mathrm{b}}$ in units of $\mathrm{K}$. Plots of $T_{\mathrm{b}}$ are shown in Fig. 10 (bottom). It can be seen at the time of the spikes at $1 \mathrm{GHz}$ $T_{\mathrm{b}}$ reaches $\sim 10^{9} \mathrm{~K}$. This is too hot to plausibly be thermal and so implies non-linear, non-thermal plasma emission. An intrinsic feature of plasma emission is a low degree of circular polarisation. Figure 10 (middle) shows that (R-L) at $1 \mathrm{GHz}$ is small in comparison to the flux $(\mathrm{R}+\mathrm{L})$ thus supporting the evidence for plasma emission at $1 \mathrm{GHz}$

Figure 11 shows plots of NoRP radio spectra following the evolution of the event beginning 01:51:00. Before this the flux at higher frequencies is still at the level of the background. The spectra are made by summing the flux over $15 \mathrm{~s}$ time intervals and normalising. Error bars at the $10 \%$ level are assigned to the data points as an estimate of errors due to atmospheric effects and local weather conditions. The spectra were then fitted using a function for gyrosynchrotron emission (Eq. (3)) allowing for four fit parameters (see http://solar.nro.nao.ac.jp/ norp/ for details): $\alpha_{\mathrm{tk}}$, the positive power index at low frequencies (corresponding to optically thick plasma), $\alpha_{\text {tn }}$ is the negative power index at high frequencies corresponding to optically thin plasma, $\hat{v}$ the turn-over frequency and $\widehat{F_{v}}$ the turn-over flux density.

$$
\begin{aligned}
F_{v} & =\widehat{F}_{v}\left(\frac{v}{\widehat{v}}\right)^{\alpha_{\mathrm{tk}}}\left\{1-\exp \left[-\left(\frac{v}{\widehat{v}}\right)^{\alpha_{\mathrm{tn}}-\alpha_{\mathrm{tk}}}\right]\right\} \\
& \approx\left\{\begin{array}{l}
\widehat{F}_{v}(v / \widehat{v})^{\alpha_{\mathrm{tk}}} \text { for } v \ll \widehat{v} \\
\widehat{F}_{v}(v / \widehat{v})^{\alpha_{\mathrm{tn}}} \text { for } v \gg \widehat{v} .
\end{array}\right.
\end{aligned}
$$




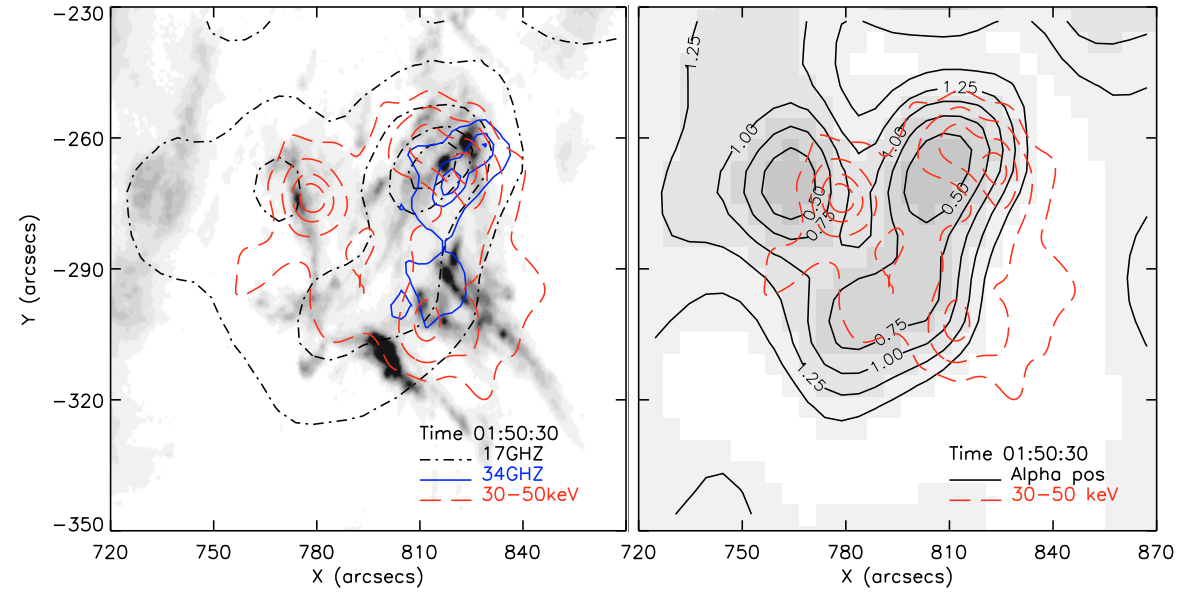

Fig. 9. Left: TRACE image with NoRH $17 \mathrm{GHz}$ (dot-dashed black), $34 \mathrm{GHz}$ (solid blue) and RHESSI 30-50 keV (dashed red) contours. Contour levels of $90,75,50 \%$ for all contours (plus $25 \%$ for $17 \mathrm{GHz}$ and $30-50 \mathrm{keV}$ ). Right: Map of radio spectral index $\alpha$ with contours of positive $\alpha$ ranging from 0 to 1.5 in steps of 0.25 (solid black) and RHESSI $30-50 \mathrm{keV}$ (dashed red).

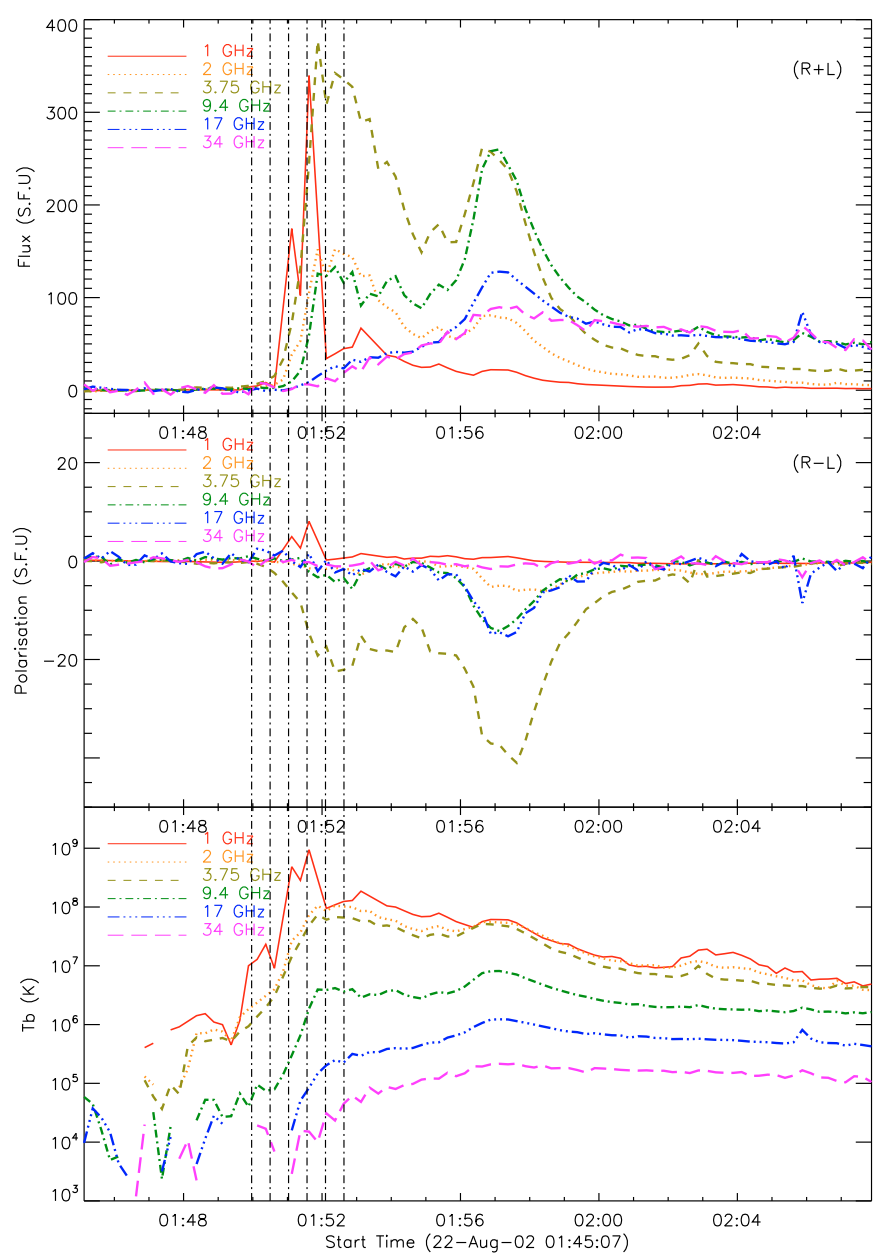

Fig. 10. Top: NoRP flux $(\mathrm{R}+\mathrm{L})$ at $1,2,3.75,9.4,17$ and $34 \mathrm{GHz}$. Middle: NoRP flux (R-L) showing degree of circular polarisation. Bottom: brightness temperature calculated at each frequency. The dashed vertical lines show time intervals over which RHESSI images were obtained for Fig. 5.

To fit the data we used the standard NoRP software which first chooses $\widehat{v}$ to be the datapoint for which there is the greatest flux, and $\widehat{F}_{v}$ is assigned this value. The parameters are then varied to find the best fit. Figure 11 shows fits to the data from 1 to $34 \mathrm{GHz}$ (solid line) and also a second fit excluding the flux at $1 \mathrm{GHz}$ (dashed line). Corresponding values of $\alpha_{\mathrm{tk}}$ and $\alpha_{\mathrm{tn}}$ for each fit are displayed on the plot, where 0 and 1 refer to the solid and dashed fits respectively. However as can be seen from the first three plots of Fig. 11, the flux peaks at $1 \mathrm{GHz}$ and the fit incorrectly tries to fit a spectrum which turns over at low frequencies. A second attempt excluding the flux at $1 \mathrm{GHz}$ provides a more acceptable fit. We conclude from this that the flux from the burst at $1 \mathrm{GHz}$ can not be described by a function for gyrosynchrotron emission and thus supports our case for the production of plasma emission at this time.

Plasma emission is at the local electron plasma frequency $\omega_{\mathrm{p}}$ or its second harmonic $2 \omega_{\mathrm{p}}$ which is dependent on the plasma density $n$. Figure 12 (http: //sunbase.nict.go.jp/ solar/denpa/hirasDB/Events/) shows a radio spectrogram from the Hiraiso Solar Observatory (HiRAS) which observes in the range 25-2500 MHz. The spectrogram shows faint type III bursts starting 01:50:50 followed by more intense bursts at $\sim 01: 51: 30$. The spectrogram shows high frequency type III bursts which have a duration of about a minute during which the frequency drifts from $\approx 1800 \mathrm{MHz}$ to $\approx 400 \mathrm{MHz}$ due to decreasing plasma density as the beam propagates out. This confirms that the bursts detected at $1 \mathrm{GHz}$ by NoRP is indeed plasma emission. At lower frequencies, more intense type III emission can be seen lasting around $4 \frac{1}{2}$ min which drifts to frequencies below the observing range of HiRAS. Figure 13 shows a spectrogram obtained from RAD2 on the WAVES experiment on the WIND satellite which observes in the range $1.075-13.825 \mathrm{MHz}$ (Bougeret et al. 1995). Figure 13 shows that the type III emission continues to drift to frequencies as low as $1 \mathrm{MHz}$. From the starting frequency of the radio bursts it is possible to determine the local plasma density $n$ and from this estimate a height at which the burst was emitted. Using $\omega_{p} \approx 1500 \mathrm{MHz}$ as an approximate starting frequency of the plasma emission, then we can estimate $n$ to be a few $10^{10} \mathrm{~cm}^{-3}$. If we assume that the type III radio burst is caused within the jet this suggests that the jet density is consistent with low coronal density.

\section{Discussion and conclusion}

The evolution of the jet observed on the 22 August 2002 is similar to that of events described by Shimojo et al. (2007) in which a twisted loop rises prior to the jet. The evolution can be compared to that described by the magnetic reconnection jet of e.g. Heyvaerts et al. (1977); Shimojo et al. (1996). Reconnection with the overlying coronal field results in field reconfiguration and the ejection of hot X-ray emitting plasma. As 


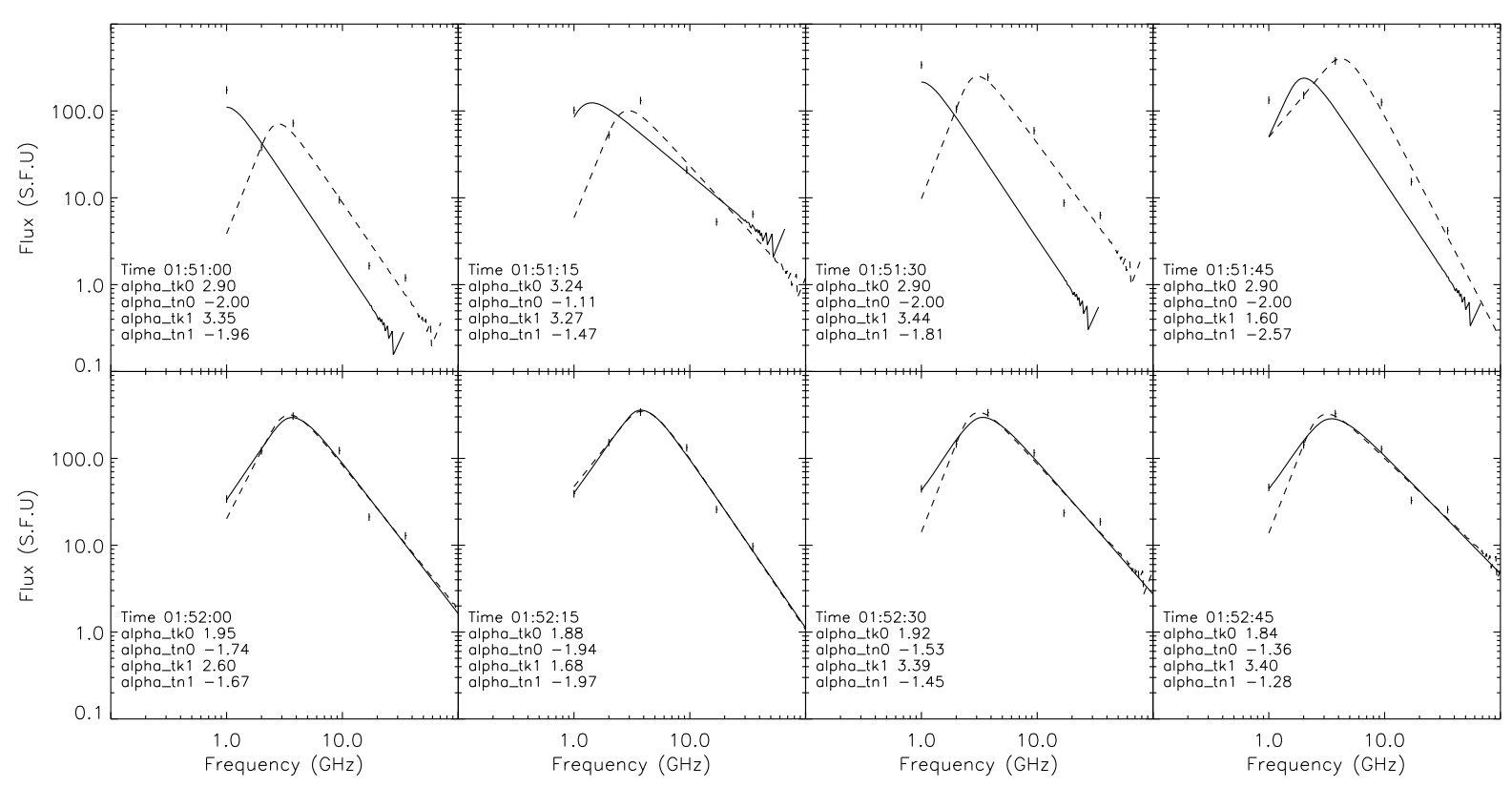

Fig. 11. NoRP radio spectra at $15 \mathrm{~s}$ time intervals. Solid line shows gyrosynchrotron function fitted to the data from 1 to $34 \mathrm{GHz}$. Dashed line shows the same function fitted to data from 2 to $34 \mathrm{GHz}$. Corresponding values of $\alpha_{t k}$ and $\alpha_{\mathrm{tn}}$ for each fit are displayed on the plot, where 0 and 1 refer to the solid and dashed fits respectively.

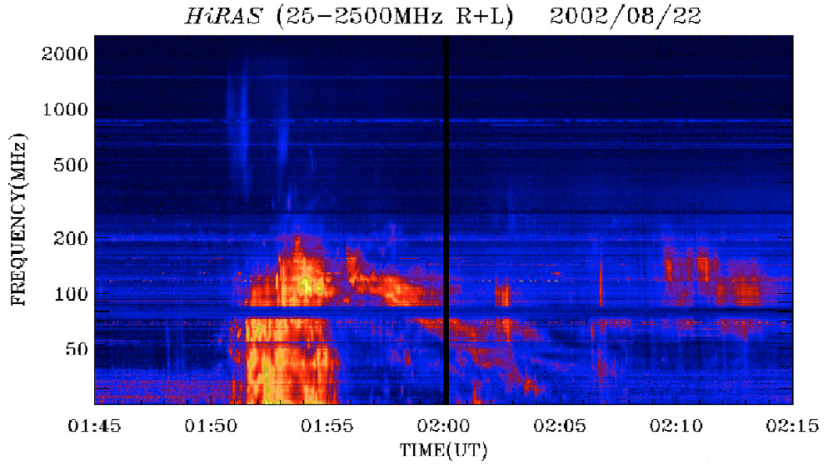

Fig. 12. Radio spectrogram from the Hiraiso solar observatory (25-2500 MHz) showing type III radio bursts. (See electronic version for colour plots.)

the event progresses TRACE observations show loops forming at the base of the jet as expected by the magnetic reconnection model. In addition to this the ejected material appears to untwist slightly as twist from the emerging field is transferred to the "open" field lines of the jet. We found an apparent jet velocity of $\sim 500 \mathrm{~km} \mathrm{~s}^{-1}$ which is consistent with predictions of velocities on the order of the Alfvén speed for this model. Calculating the Alfvén speed, $v_{\mathrm{A}}=B /(4 \pi \rho)^{1 / 2}$ for $B=10 \mathrm{G}$ and $n=10^{10} \mathrm{~cm}^{-3}$ we obtain $v_{\mathrm{A}} \approx 200 \mathrm{~km} \mathrm{~s}^{-1}$. However it should be noted that for plasma temperatures such as those found from RHESSI spectroscopy the sound speed in the corona will be in the range of $640-780 \mathrm{~km} \mathrm{~s}^{-1}$.

At the time of the main ejection RHESSI imaging shows hard X-rays to as high as $30-50 \mathrm{keV}$ present in the jet. This is the first time that hard X-rays coming from the jet location have been reported. Sui et al. (2006) have reported an event which may demonstrate hard X-ray emission from a jet region, a 6-12 keV coronal source was observed in the vicinity of a cusp-like, possibly jetting, structure above the apex of a compact flare loop.
However without imaging spectroscopy it was not possible to determine if the source was thermal or non-thermal. Krucker et al. (2008) found evidence for non-thermal coronal X-rays in the 14-30 keV range at the onset time of an interplanetary type III radio burst, suggesting emission by escaping fast electrons, however there was no radio or other coronal imaging of a jet or other feature supporting this interpretation. By contrast, our observations show a clear spatial as well as temporal association of the jet with the non-thermal emission in both radio and hard X-rays, and therefore we believe this to be the first clear observation of non-thermal emission from flare-accelerated escaping electrons. RHESSI imaging spectroscopy includes a broken power law fit function which yields a spectral index of $\sim 4.5$ confirming the presence of non-thermal jet electrons.

If the non-thermal emission is bremsstrahlung caused by electrons propagating in a thick target coronal source in the jet then we can estimate the number of electrons above $20 \mathrm{keV}$ using Eqs. (4), (5) and (6)

$$
\begin{aligned}
& F\left(E>E_{\mathrm{c}}\right)=\int_{E_{\mathrm{c}}}^{\infty} f_{\mathrm{e}}(E) \mathrm{d} E \\
& f_{\mathrm{e}}(E)=3.28 \times 10^{33} \frac{b(\gamma)}{\gamma} A E_{\mathrm{c}}^{-\gamma} \\
& b(\gamma)=\gamma^{2}(\gamma-1)^{2} B\left(\gamma-\frac{1}{2}, \frac{3}{2}\right) \approx 0.27 \gamma^{3}
\end{aligned}
$$

where $f_{\mathrm{e}}(E)$ (electrons $\mathrm{keV}^{-1} \mathrm{~s}^{-1}$ ) is the electron injection spectrum, $E_{\mathrm{c}}$ is the electron energy cutoff, $B$ is the Beta function, and $A$ is the photon flux at $1 \mathrm{keV}$ determined by fitting a power law to the photon spectrum where $I\left(\epsilon_{x}\right)=A \epsilon_{x}^{-\gamma}$ (Brown 1971; Hudson et al. 1978). We find this to be $1.5 \times 10^{35}$ electrons s ${ }^{-1}$. The thick target model is the most efficient method of converting energy from the electrons into photons (apart from thermal bremsstrahlung emission in a confined source). Taking a jet length of $40^{\prime \prime}\left(2.9 \times 10^{9} \mathrm{~cm}\right)$ suggests a total column depth of $\sim 2.9 \times 10^{19} \mathrm{~cm}^{-2}$, which is collisionally thick to electrons 


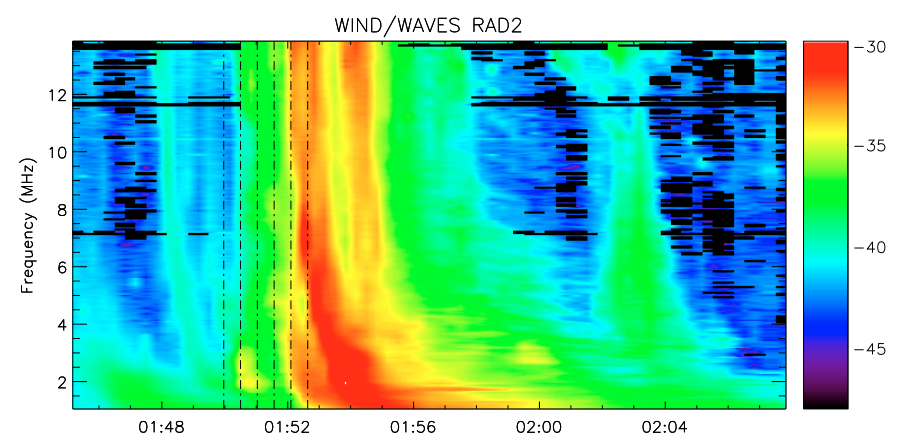

Fig. 13. Spectrogram from WAVES on the WIND spacecraft (1.075-13.825 MHz) showing type III radio bursts.

of $10 \mathrm{keV}$. Therefore we consider also a hard X-ray emitting thin target through which the electrons are being ejected. From Eqs. (4), (7) and (8)

$f_{\mathrm{e}}(E)=1.05 \times 10^{42} C(\gamma) A \frac{1}{n_{0} E^{-(\gamma-1 / 2)}}$

$C(\gamma)=\frac{(\gamma-1)}{B\left(\gamma-1, \frac{1}{2}\right)} \approx(\gamma-1.5)^{1.2}$

where $f_{\mathrm{e}}(E)$ (electrons $\left.\mathrm{keV}^{-1}\right)$ is the thin target electron spectrum and $n_{0}$ is the target density. We estimate the instantaneous number of radiating electrons to be $1 \times 10^{35}$ electrons. An electron of $30 \mathrm{keV}$ will have a residence time of $0.29 \mathrm{~s}$ in the jet. For a thin target interpretation the number of ejected electrons is then $3 \times 10^{35}$ electrons s $^{-1}$.

In addition, RHESSI spectral fitting suggests a jet temperature of $\sim 28 \mathrm{MK}$. As no hot loops are seen in this region with TRACE at this time or slightly after we are confident that this indeed the temperature of the jet. As suggested by Feldman et al. (1999), at these high temperatures the thermal continuum from free-free and free-bound emission will be significant. From this it is plausible to suggest that TRACE images reveal free-free emission in the jet similar to that observed with RHESSI. NoRH observations at 17 and $34 \mathrm{GHz}$ are seen to be co-spatial with the hard X-ray emission during this time and $\alpha$ is found to be $\sim 0.75$ corresponding to optically thick emission by non-thermal electrons, reinforcing our claim that that X-rays are directly revealing the presence of these particles in the jet. Further radio observations from NoRP and HiRAS show simultaneous bursts of plasma emission at $1 \mathrm{GHz}$. If we assume this results from a beam of electrons accelerated in the jet, we calculate $n=10^{10} \mathrm{~cm}^{-3}$ which is a reasonable jet density. We believe this to be a rare observation, but one lending strong support to a model involving coronal electron acceleration in a relatively dense plasma and in close association with the magnetic reconfiguration that launches the jet.

Acknowledgements. H.M.B. gratefully acknowledges the support of an STFC studentship. L.F. gratefully acknowledges the support of an STFC Rolling Grant, and financial support by the European Commission through the SOLAIRE Network (MTRN-CT_2006-035484). We would like to thank Brian Dennis for useful comments regarding RHESSI spectral fitting. We would like to also thank NoRH and NoRP teams for help regarding radio analysis, and an anonymous referee whose suggestions helped us to improve this paper.

\section{References}

Alexander, D., \& Fletcher, L. 1999, Sol. Phys., 190, 167

Aschwanden, M. J., Nightingale, R. W., Tarbell, T. D., \& Wolfson, C. J. 2000, Astrophys. J., 535, 1027

Bougeret, J.-L., Kaiser, M. L., Kellogg, P. J., et al. 1995, Space Sci. Rev., 71, 231

Brown, J. C. 1971, Sol. Phys., 18, 489

Canfield, R. C., Reardon, K. P., Leka, K. D., et al. 1996, ApJ, 464, 1016

Chifor, C., Young, P. R., Isobe, H., et al. 2008, A\&A, 481, L57

Christe, S., Krucker, S., \& Lin, R. P. 2008, ApJ, 680, L149

Dulk, G. A. 1985, ARA\&A, 23, 169

Feldman, U., Laming, J. M., Doschek, G. A., Warren, H. P., \& Golub, L. 1999, ApJ, 511, L61

Golub, L., Deluca, E., Austin, G., et al. 2007, Sol. Phys., 243, 63

Handy, B. N., Acton, L. W., Kankelborg, C. C., et al. 1999, Sol. Phys., 187, 229 Heyvaerts, J., Priest, E. R., \& Rust, D. M. 1977, ApJ, 216, 123

Hudson, H. S., Canfield, R. C., \& Kane, S. R. 1978, Sol. Phys., 60, 137

Krucker, S., \& Lin, R. P. 2002, Sol. Phys., 210, 229

Krucker, S., Saint-Hilaire, P., Christe, S., et al. 2008, ApJ, 681, 644

Lin, R. P., Dennis, B. R., et al. 2002, Sol. Phys., 210, 3

Moreno-Insertis, F., Galsgaard, K., \& Ugarte-Urra, I. 2008, ApJ, 673, L211

Nakajima, H., Nishio, M., Enome, S., et al. 1994, IEEE Proc., 82, 705

Nishizuka, N., Shimizu, M., Nakamura, T., et al. 2008, ApJ, 683, L83

Pariat, E., Antiochos, S. K., \& DeVore, C. R. 2009, ApJ, 691, 61

Savcheva, A., Cirtain, J., Deluca, E. E., et al. 2007, PASJ, 59, 771

Shibata, K., \& Uchida, Y. 1986, Sol. Phys., 103, 299

Shibata, K., Ishido, Y., Acton, L. W., et al. 1992, PASJ, 44, L173

Shibata, K., Nitta, N., Strong, K. T., et al. 1994, ApJ, 431, L51

Shimojo, M., \& Shibata, K. 2000, ApJ, 542, 1100

Shimojo, M., Hashimoto, S., Shibata, K., et al. 1996, PASJ, 48, 123

Shimojo, M., Narukage, N., Kano, R., et al. 2007, PASJ, 59, 745

Sterling, A. C., Shibata, K., \& Mariska, J. T. 1993, ApJ, 407, 778

Strong, K. T., Harvey, K., Hirayama, T., et al. 1992, PASJ, 44, L161

Sui, L., Holman, G. D., \& Dennis, B. R. 2006, ApJ, 646, 605

Torii, C., Tsukiji, Y., Kobayashi, S., et al. 1979, Nagoya University, Research Institute of Atmospherics, Proceedings, 26, 129

Török, T., \& Kliem, B. 2005, ApJ, 630, L97

Tsuneta, S., Acton, L., Bruner, M., et al. 1991, Sol. Phys., 136, 37

Uchida, Y. 1969, PASJ, 21, 128

Yokoyama, T., \& Shibata, K. 1995, Nature, 375, 42 